\title{
Evaluation of the criterion and convergent validity of the Diagnostic Interview for Social and Communication Disorders in young and low-functioning children
}

\author{
Jarymke Maljaars ${ }^{1}$, Ilse Noens ${ }^{2}$, Evert Scholte ${ }^{1}$ \& Ina van Berckelaer-Onnes ${ }^{1}$ \\ ${ }^{1}$ Leiden University, the Netherlands; ${ }^{2}$ Katholieke Universiteit Leuven, Belgium
}

\begin{abstract}
The Diagnostic Interview for Social and Communication Disorders (DISCO; Wing, 2006) is a standardized, semi-structured and interviewer-based schedule for diagnosis of autism spectrum disorder (ASD). The objective of this study was to evaluate the criterion and convergent validity of the DISCO-11 ICD-10 algorithm in young and low-functioning children. The DISCO-11, Autism Diagnostic Observation Schedule (ADOS), and Social Communication Questionnaire (SCQ) were administered to a Dutch sample of 115 children comprising 52 children with ASD (both with and without intellectual disability), 26 children with intellectual disability (non-ASD), and 37 typically developing children. Results indicated high sensitivity and specificity for DISCO-11 classifications in differentiating ASD from non-ASD according to the clinical classification in children with mild intellectual disability or average intelligence. Among children with a moderate or severe intellectual disability the sensitivity was equally high, but the specificity was significantly lower. The agreement between DISCO-11 and ADOS classifications was substantial, between DISCO-11 and SCQ moderate. The correlations between raw scores of the DISCO and ADOS algorithm or SCO were both high. In conclusion, the DISCO-11 differentiates accurately between autistic disorder and non-ASD in young children with an average intelligence or mild intellectual disability, but is over inclusive in the lower levels of intellectual disability.
\end{abstract}

Keywords: autism spectrum disorder, DISCO-11, diagnostic interview, validity

\section{Introduction}

A substantial proportion of individuals with autism spectrum disorder (ASD) also have an intellectual disability (ID). Reported percentages are variable (ranging from about $15 \%$ to $70 \%$; Bhaumik et al., 2010), depending on diagnostic criteria and level of functioning. The diagnosis of ASD in ID can be complicated, especially in those with severe or profound ID (Bhaumik et al., 2010; Vig \& Jedrysek, 1999). Nevertheless, diagnosis of ASD in ID is important in order to adjust treatment strategies and to provide access to specific services. A detailed interview with the parents about developmental history and current behavior is an essential part of the assessment procedure to diagnose ASD (Bishop et al., 2008; Filipek et al., 1999). Therefore, it is necessary to examine the applicability of standardized interviews in the ID population.

The Diagnostic Interview for Social and Communication Disorders - version 11 (DISCO-11) is a semi-structured interview to be administered with parents or other primary carers (Wing, 2006). The instrument can be used to assess individual needs and to diagnose ASD and is suitable for individuals of all ages, levels of abilities, and the whole spectrum (for a more detailed description of the DISCO, see Wing et al., 2002). The DISCO is constructed in a dimensional rather than a categorical way, as a bottom-up approach was used to compose the items. Based on the broad- 
ranging nature of ASDs, the DISCO items cover a very wide range of skills and features. Originally, the DISCO was designed for clinical use to provide a comprehensive description of individual characteristics and needs, but the interview can also be used for classification in clinical practice or for research. For this purpose, computerized algorithms have been developed. These algorithms provide classifications based on official classification systems, such as the ICD-10, and other specific diagnostic categories (Wing et al., 2002). The algorithms for ICD-10 Childhood Autism and Atypical Autism are based on a selection of 93 DISCO items and were used in this study.

Thus far, the psychometric properties of the DISCO (versions 9 and 10) have only been tested in the United Kingdom and Sweden. With regard to the reliability, the inter-rater reliability proved to be moderate to good or excellent for the algorithm items (Leekam et al., 2002; Nygren et al., 2009), but also for the other items (Wing et al., 2002). As for validity, good agreement across clinical diagnoses and the DISCO algorithms for ICD-10 $(\kappa=.57)$ and Wing and Gould ASD $(\kappa=.79)$ has been demonstrated (Leekam et al., 2002). Nygren et al. (2009) also found a good agreement (89\%) between the DISCO and ADI-R classification based on 57 interviews. Furthermore, age, nonverbal IQ, language production and comprehension were not significantly related to the DISCO classifications (Leekam et al., 2002).

As far as we know, studies into the psychometric properties of the DISCO are limited to the three aforementioned studies. No reports have focused specifically on young and low-functioning children. Since the DISCO was designed for any age and any level of ability (Wing et al., 2002), the DISCO may be useful for autism diagnoses in ID. Therefore, the aim of this study was to evaluate the criterion and convergent validity of the DISCO ICD-10 algorithms in young and low-functioning children. To this end, first the sensitivity and specificity of the DISCO ICD-10 classifications were explored, and second, the DISCO results were compared to the ADOS and SCO outcomes.

\section{Method}

\section{Participants}

The sample for this study comprised three different subgroups: children with ASD, children with ID, and typically developing children (TD) (see Table 2.1).

Table 2.1 Main characteristics of the participants

\begin{tabular}{|c|c|c|c|c|c|c|c|c|c|}
\hline & \multicolumn{3}{|c|}{$\operatorname{ASD}(n=52)$} & \multicolumn{3}{|c|}{ ID (non-ASD) $(n=26)$} & \multicolumn{3}{|c|}{$\mathbf{T D}(n=37)$} \\
\hline & Range & $M$ & $S D$ & Range & $M$ & $S D$ & Range & $M$ & $S D$ \\
\hline Chronological age $^{\text {a }}$ & $34-137$ & 79.6 & 28.9 & $48-134$ & 80.9 & 20.7 & $24-49$ & 37.9 & 6.3 \\
\hline Nonverbal mental age ${ }^{a}$ & $<24^{b}-70$ & 43.0 & 14.2 & $<24^{b}-63$ & 38.2 & 12.5 & $29-65$ & 42.5 & 9.2 \\
\hline Receptive language ${ }^{\text {a }}$ & $<14^{b}-65$ & 33.9 & 15.8 & $<14^{b}-70$ & 37.3 & 13.8 & $22-75$ & 44.3 & 11.1 \\
\hline \multirow[t]{2}{*}{ Expressive language ${ }^{a}$} & $<18^{b}-75$ & 37.6 & 16.4 & $<18^{b}-53$ & 34.7 & 11.9 & $22-72$ & 43.6 & 9.5 \\
\hline & $n$ & \multicolumn{2}{|c|}{$\%$} & $n$ & \multicolumn{2}{|c|}{$\%$} & $n$ & \multicolumn{2}{|c|}{$\%$} \\
\hline \multicolumn{10}{|l|}{ Gender } \\
\hline Male & 43 & \multirow{2}{*}{\multicolumn{2}{|c|}{$\begin{array}{l}83 \% \\
17 \%\end{array}$}} & 16 & \multicolumn{2}{|c|}{$61 \%$} & 15 & \multicolumn{2}{|c|}{$41 \%$} \\
\hline Female & 9 & & & 10 & & & 22 & & \\
\hline \multicolumn{10}{|l|}{ Level of ID } \\
\hline No ID & 11 & \multicolumn{2}{|c|}{$15 \%$} & 0 & \multicolumn{2}{|c|}{-} & 37 & \multicolumn{2}{|c|}{$100 \%$} \\
\hline Borderline ID & 6 & \multicolumn{2}{|c|}{$23 \%$} & 0 & \multicolumn{2}{|c|}{ - } & 0 & \multicolumn{2}{|c|}{ - } \\
\hline Mild ID & 15 & \multicolumn{2}{|c|}{$29 \%$} & 11 & \multicolumn{2}{|c|}{$42 \%$} & 0 & \multicolumn{2}{|c|}{ - } \\
\hline Moderate ID & 12 & \multicolumn{2}{|c|}{$12 \%$} & 12 & \multicolumn{2}{|c|}{$46 \%$} & 0 & \multicolumn{2}{|c|}{-} \\
\hline Severe ID & 8 & \multicolumn{2}{|c|}{$21 \%$} & 3 & \multicolumn{2}{|c|}{$12 \%$} & 0 & \multicolumn{2}{|c|}{ - } \\
\hline
\end{tabular}


Note. $\mathrm{ASD}=$ autism spectrum disorder; ID = intellectual disability; TD = typical development

a age / age equivalent in months; ${ }^{\mathrm{b}}$ minimum score

All children were less than twelve years old and had a developmental age below six years. The ASD group ( $n=52$ ) mainly consisted of boys and the majority had an associated ID. All children with ASD had received an independent clinical diagnosis by qualified professionals before participation in this study (see Procedure). These were mainly children with a clinical classification of autistic disorder. The ID group consisted of 26 children. These were children with either known causes of ID, such as Down syndrome, or unknown causes, but all without ASD. The level of ID ranged from mild to severe. About half of the children had associated conditions, such as ADHD or language disorder. The TD group contained 37 children between 2 and 4 years old.

\section{Instruments}

Diagnostic Interview for Social and Communication Disorders. The DISCO-11 can be administered to collect information about developmental history and description of a broad range of skills and behaviors (Wing, 2006; Wing et al., 2002). The Dutch translation of the $11^{\text {th }}$ version of the interview was used in this study (Van Berckelaer-Onnes et al., 2008). The interview takes about two to three hours to administer and consists of more than 300 questions grouped under different sections: 'Family, medical background and identifying information', 'Infancy', 'Developmental skills', 'Repetitive, stereotyped activities', 'Emotions', 'Maladaptive behavior', and 'Interviewer's judgment of quality'. The sections concerning psychiatric illnesses, difficulties related to sexual behavior, and forensic problems were not used in this study, because they were not relevant for young and lowfunctioning children. The majority of the items are scored in three levels of severity: 'marked problem', 'minor problem', or 'no problem'. Furthermore, the DISCO distinguishes both 'ever' and 'current' ratings of the child's behavior. In this way, a distinction is made between current symptoms and behavior that has ever been present. For this study, the ICD-10 classifications Childhood Autism and Atypical Autism were collapsed together to compose an ASD or non-ASD classification. Furthermore, only 'current' classifications were used, because no differences were found between current and ever results.

Autism Diagnostic Observation Schedule. The Autism Diagnostic Observation Schedule (ADOS) is a semi-structured, standardized assessment in which standardized toys and activities are used to provide opportunities to elicit social, communicative, play, and stereotyped behaviors relevant to the diagnosis of ASD (Lord et al., 1999). Each child was administered activities from module 1 or 2 based on the expressive language level by officially trained examiners. The revised algorithms and cut-offs were used to provide ASD and non-ASD classifications (Gotham et al., 2007). Inter-rater and test-retest reliability, as well as validity, have been demonstrated (e.g. Gotham et al., 2007, 2008; Lord et al., 2000). Validity of the ADOS was also examined in the ID population (De Bildt et al., 2004).

Social Communication Questionnaire. The Social Communication Questionnaire (SCQ; Rutter et al., 2003; Dutch translation: Warreyn et al., 2004) is a parent-report questionnaire with 40 binary-scaled items to screen for ASD based on the Autism Diagnostic Interview - Revised (ADI-R; Lord et al., 1994). The recommended cut-off score for ASD is 15 (Rutter et al., 2003), and proved to be accurate in the ID population (Witwer \& Lecavalier, 2007). The SCQ is available in two forms: lifetime and 
current. In this study the 'current' version which evaluates the child's behavior over the most recent 3 months was used. This version is also suitable for children under the age of 5 years. The SCO has been found reliable (Rutter et al., 2003) and valid by several studies (e.g. Berument et al., 1999; Chandler et al., 2007). Reliability and validity of the Dutch translation have been tested in Belgium (Warreyn et al., 2004).

Other instruments. To measure nonverbal mental age a Dutch test for nonverbal intelligence, the SON-R 21/2-7 (Tellegen et al., 1998) was used. Language abilities were individually assessed using the Reynell test for Dutch language comprehension (Van Eldik et al., 1995) and the Schlichting test for Dutch language production (Schlichting et al., 1995). Raw scores were converted into age equivalents. All three instruments have been found reliable and valid by the authors and the Committee for Psychometric Evaluation of Tests of the Dutch Institute of Psychologists (Evers et al., 2009).

\section{Procedure}

Families of children with ASD and/or ID were contacted through 23 special schools and day care centers. The professionals involved were asked to include children between 2 and 12 years old with an official ASD diagnosis or children without ASD, and to exclude children without official diagnosis. Severe sensory or severe motor impairments were exclusion criteria for all groups, because several items from the DISCO, ADOS, and SCO are difficult to code in these cases. TD children were recruited from four regular day care centers. All regular and special schools and day care centers were randomly selected from schools and day care centers situated in three different regions of the Netherlands (Zuid-Holland, Noord-Holland, and Utrecht). All ASD children were clinically diagnosed based on DSM-IV-TR criteria for pervasive developmental disorders prior to recruitment for the study by independent and qualified professionals. The DISCO was not used and none of the researchers of the current study were involved in the diagnostic procedure. The time gap between previous diagnosis and participation in this study varied between one and seven years. Parents were asked to send a copy of the diagnostic report from the child's clinical record. Informed consent was obtained from all parents. The original sample consisted of 129 cases, from which 14 children were excluded, because the clinical diagnosis of the child was unclear or the DISCO could not be administered due to refusal or insufficient Dutch language abilities of the parents. Eventually, 115 parents and children participated in this study. Comparisons to the ADOS were based on 109 cases and comparisons to the SCO on 106 cases.

The DISCO interviews were conducted by 7 different interview couples at the parents' home. The interviewers were trained to use the DISCO specifically for the purpose of the study. In most of the cases they did not see the child before or during the interview. The interviewers and raters were blind to the previous diagnosis before the DISCO interview. The ADOS was administered at the university or at school in presence of one of the parents. The study was designed so that different researchers administered the interview and the ADOS. The parents filled out the Social Communication Questionnaire at home. The SON-R 21/2-7, Reynell, and Schlichting were administered at the child's school or day care.

\section{Data analyses}

First, raw scores of the three subgroups on total and domain scores of the ICD-10 algorithm were compared to examine whether the DISCO differentiates between ASD and non-ASD cases. The 
domains Social Impairment, Communicative Impairment, and Repetitive Behavior and Activities are based on 38, 15, and 29 items, respectively. The raw scores were calculated by summing the number of recorded symptoms for each domain. The rating code that is demanded in the algorithm in order to count as positive for the relevant diagnostic criterion was used. Most of the DISCO items are coded as severe (0), minor (1), or no problem (2). To meet the criteria for a symptom in specific domain, a 'severe' code is needed in most of the cases. Differences in mean scores between the three groups were evaluated by conducting several analyses of variance followed by post hoc tests. The Games-Howell procedure was used, because Levene's tests reported unequal variances between the groups. Variances in the ASD group were higher than in the ID and TD groups. Second, sensitivity and specificity rates, positive and negative predictive values were computed for the DISCO, ADOS, and SCO compared to the clinical diagnoses. Third, agreement between the different autism instruments was examined by computing Kappas on the classification outcomes and correlations on the raw scores.

\section{Results}

\section{DISCO ICD-10 algorithm scores}

The mean raw scores on the different subcategories of the DISCO ICD-10 algorithm for the three different subgroups are presented in Table 2.2 .

Table 2.2 Description and comparisons of raw DISCO ICD-10 algorithms scores for the subgroups

\begin{tabular}{|c|c|c|c|c|c|c|c|c|c|c|}
\hline \multirow[b]{2}{*}{ DISCO $^{a}$} & \multicolumn{2}{|c|}{$\operatorname{ASD}(n=52)$} & \multicolumn{2}{|c|}{ ID $(n=26)$} & \multicolumn{2}{|c|}{ TD $(n=37)$} & \multicolumn{3}{|c|}{ ANOVA } & \multirow{2}{*}{$\begin{array}{c}\text { Games-Howell } \\
\text { post-hoc differences }\end{array}$} \\
\hline & $M$ & $S D$ & $M$ & $S D$ & $M$ & $S D$ & $F$ & $p$ & $\eta^{2}$ & \\
\hline SOC & 17.9 & 6.0 & 6.4 & 4.0 & 2.4 & 1.4 & 139.8 & $<.001$ & .71 & $A S D>I D>T D$ \\
\hline COM & 6.0 & 2.3 & 1.7 & 1.5 & 0.2 & 0.5 & 134.3 & $<.001$ & .71 & $A S D>I D>T D$ \\
\hline RRB & 6.0 & 3.8 & 1.6 & 2.2 & 0.2 & 0.8 & 52.3 & $<.001$ & .48 & $A S D>I D>T D$ \\
\hline Total & 29.9 & 9.5 & 9.7 & 6.0 & 2.8 & 1.9 & 172.9 & $<.001$ & .76 & ASD $>I D>T D$ \\
\hline
\end{tabular}

Note. $\mathrm{SOC}=$ social impairment; $\mathrm{COM}=$ communication impairment; $\mathrm{RRB}=$ restricted repetitive behaviors / activities ${ }^{a}$ scores were based on number of recorded symptoms for each domain

Significant differences were found between all three subgroups on the different domains and the total algorithm score $(p<.001$, large effect sizes). Post-hoc test revealed that for all domains the ASD group obtained significantly higher mean raw scores compared to both the ID and TD groups. Furthermore, the ID group scored significantly higher on all domains than the TD group (see Table 2.2).

\section{Sensitivity and specificity}

Sensitivity and specificity rates of the DISCO and the other autism instruments were computed (see Tables 2.3 and 2.4). For the DISCO, a sensitivity of .96 and a specificity of .79 were found. The specificity of the DISCO in relation to the ID group was much lower than in relation to the TD group. The sensitivity of the ADOS was comparable to the DISCO, but the SCQ led to much more false negative classifications and therefore to a lower sensitivity. The specificity for both the ADOS and SCO was higher than for the DISCO, although $95 \%$ confidence intervals of the three tests overlap to some extent. 
Table 2.3 Sensitivity, specificity, PPV and NPV for the DISCO, ADOS, and SCQ

\begin{tabular}{lrccccccc}
\hline Instruments & Sample & $n$ & Sens & $95 \% \mathrm{Cl}$ & Spec & $95 \% \mathrm{Cl}$ & PPV & NPV \\
\hline DISCO & Total sample & 115 & .96 & $.86-.99$ & .79 & $.67-.88$ & .79 & .96 \\
(ICD-10 algorithm) & ASD+TD & 89 & .96 & $.86-.99$ & 1.00 & $.88-1.00$ & 1.00 & .95 \\
& ASD+ID & 78 & .96 & $.86-.99$ & .50 & $.29-.68$ & .79 & .87 \\
\hline ADOS & Total sample & 109 & .92 & $.81-.98$ & .95 & $.84-.99$ & .94 & .93 \\
(cut-off: ASD) & ASD+TD & 85 & .92 & $.81-.98$ & .97 & $.82-1.00$ & .94 & .93 \\
& ASD+ID & 76 & .92 & $.81-.98$ & .92 & $.72-.99$ & .96 & .85 \\
\hline SCQ & Total sample & 106 & .67 & $.51-.79$ & .93 & $.82-.98$ & .89 & .78 \\
(cut-off: 15) & ASD+TD & 84 & .67 & $.51-.79$ & 1.00 & $.87-1.00$ & 1.00 & .69 \\
& ASD+ID & 70 & .67 & $.51-.79$ & .82 & $.59-.94$ & .89 & .53 \\
\hline
\end{tabular}

Note. Sens = sensitivity; Spec = specificity; PPV = positive predictive value; NPV = negative predictive value

When the DISCO classifications ASD or non-ASD were compared to the clinical diagnosis, the majority of the children were correctly classified as having ASD $(n=50)$ or no ASD $(n=50)$. Thirteen false positive cases and two false negative cases were identified. All of the 13 false positive cases had ID, thus no TD children were present in this category.

Table 2.4 Cross tab comparing DISCO-11 classifications to clinical diagnoses

\begin{tabular}{|c|c|c|c|c|c|c|}
\hline & & \multirow[b]{2}{*}{$n$} & \multicolumn{4}{|c|}{ Clinical dx ASD } \\
\hline & & & & Absent & \multirow{2}{*}{$\frac{\text { Present }}{2}$} & \multirow{2}{*}{$\frac{\text { Totals }}{52}$} \\
\hline DISCO-11 & Total sample & 115 & Test negative & 50 & & \\
\hline \multirow[t]{8}{*}{ (ICD-10 algorithm) } & & & Test positive & 13 & 50 & 63 \\
\hline & & & Totals & 63 & 52 & 115 \\
\hline & ASD+TD & 89 & Test negative & 37 & 2 & 39 \\
\hline & & & Test positive & 0 & 50 & 50 \\
\hline & & & Totals & 37 & 52 & 89 \\
\hline & ASD+ID & 78 & Test negative & 13 & 2 & 15 \\
\hline & & & Test positive & 13 & 50 & 63 \\
\hline & & & Totals & 26 & 52 & 78 \\
\hline
\end{tabular}

When the false positives $(n=13)$ and true negatives $(n=13)$ in the ID group were compared, no significant differences in gender $\left(\chi_{(1)}^{2}=0.01, p=.99\right)$, age $(U=72.0, p=.55)$, language abilities ( $U$ $=38.0, p=.09)$, associated conditions $\left(\chi_{(1)}^{2}=2.5, p=.12\right)$, or interviewers $\left(\chi_{(4)}^{2}=3.7, p=.45\right)$ were observed. However, these analyses were based on very small sample sizes. The most obvious difference was seen in severity of ID $\left(\chi_{(1)}^{2}=12.8, p<.001\right)$. The false positive group mainly consisted of children with a severe or moderate level of ID $(92 \%)$, while most of the children in the true negative group had only a mild ID (77\%).

\section{Comparison to ADOS and SCO}

Kappas were computed as a measure of agreement between classifications on the three autism instruments. The agreement between DISCO and ADOS classifications was substantial $(\kappa=.69, p<$ .001). However, in comparison to the SCO a much lower Kappa and only a moderate agreement was found ( $k=.49, p<.001$ ). The SCQ had more false negatives and fewer false positives, in other words the opposite pattern of the DISCO classifications, which explains the lower Kappa. The Kappas for the ID group were much lower than for the TD group for both instruments. 
The correlations between raw total scores of the DISCO algorithm with the ADOS $\left(r_{(107)}=.87\right.$, $p<.001)$ and SCO $\left(r_{(104)}=.86, p<.001\right)$ were both high. Regarding the domains, the correlation between DISCO and ADOS social / communication domain scores $\left(r_{(107)}=.87, p<.001\right)$ was much higher than between the restricted / repetitive behavior domain scores $\left(r_{(107)}=.64, p<.001\right)$, but were both significant.

\section{Discussion}

The aim of the current study was to evaluate the criterion and convergent validity of the DISCO-11 ICD-10 algorithms in young and low-functioning children. Overall, the sensitivity rate was high, the specificity rate was acceptable, and the agreement with the ADOS and SCO was sufficient. The results support the utility of the DISCO as an effective diagnostic tool for ASD in children with average intelligence or mild ID, since the results of the current study indicated a good criterionrelated and convergent validity. However, among the individuals with moderate to severe levels of ID, the specificity was significantly lower than among individuals with a mild ID to an average level of functioning. In other words, the DISCO accurately identifies the low-functioning children with ASD, but is over inclusive. The DISCO classifications were more accurate at a mild level of ID. These findings were in line with previous studies (Leekam et al., 2002; Posserud et al., 2009).

The agreement between DISCO-11 and ADOS classifications was substantial. Convergent validity was examined by calculating the relationship between the raw scores on the DISCO-11 and ADOS. In particular, the social and communication domain scores were strongly related. The correlation between restricted and repetitive behavior domain scores was weaker, but still satisfactory. The ADOS only contains three items in the restricted and repetitive behavior domain, whereas the DISCO score is based on 29 items and covers a broader range of repetitive behaviors and activities. The correlation between the raw scores on the DISCO and SCO was comparable to the correlation with the ADOS, whereas the agreement between the DISCO and SCO classifications was much lower. Probably the cut-off score of the SCO is not accurate for this young and lowfunctioning group. It is important to notice that the SCQ is primarily designed for children of 4 years and older, and most of the children in this study were younger or lower functioning. The study of Witwer and Lecavalier (2007) indicated a cut-off score of 15 as accurate for individuals with ID. However, recent studies in young children ( $<5$ years) showed that maximum sensitivity and specificity rates were achieved when the cut-off score was reduced (e.g., Corsello et al., 2007; Snow \& Lecavalier, 2008; Wiggins et al., 2007). The data of our study support the latter findings. Again, the results indicated a lower convergent validity of the DISCO-11 in individuals with ID compared to individuals with an average intelligence.

Severity of ID often has a disturbing influence on the proper classification of diagnostic instruments. Several other diagnostic and screening instruments for ASD were also found to be over inclusive in the severe levels of ID (e.g., De Bildt et al., 2003, 2004a; Gray et al., 2008; Witwer \& Lecavalier, 2007), because it is difficult to determine whether specific behaviors are associated with ID or ASD. Some features, such as absence of language development and imagination, can be a consequence of a delay or low level of functioning, instead of an impaired development as seen in ASD (Bhaumik et al., 2010). When using the DISCO for very low-functioning children, some of the above mentioned features were recorded as ASD symptoms, without taking the general level of functioning into account. Therefore, a thorough examination of the intellectual and adaptive level of 
functioning with reliable and valid instruments is always necessary. These results underline the necessity of a comprehensive assessment by experienced clinicians to diagnose ASD.

Some limitations of the current study have to be mentioned. First, the sample size was relatively modest, in particular when specific subsamples were examined. Second, this study was not primarily designed as a validation study. In most of the cases the interviewers did not see the child before or during the interview, but in some cases the child was present at home during the interview. The interviewers and raters were blind to the previous diagnosis before the DISCO interview. However, some parents referred to the diagnosis during the conversation, which may have biased the outcomes. Third, all children with ASD were previously clinically diagnosed; as a result, parents' answers might have been influenced by familiarity with the types of questions and the knowledge of their child's diagnosis. And last, the ID group possibly contains undiagnosed ASD cases.

Given the influence of severity of ID on the classification, a more specific examination of the ICD-10 algorithm in different levels of intellectual functioning would be important in further research. The data of our study suggest that there are differences in raw scores between ASD and non-ASD, also at the lower levels of ID. Therefore, the possibilities for adjusted criteria can be explored. Since the majority of the participants received a clinical diagnosis of autistic disorder, the accurateness of the DISCO ICD-10 algorithm for broader ASDs in ID still has to be evaluated. This study and previous studies mainly focused on children, and therefore the psychometric properties for adults with ID also need attention in further research. Furthermore, only the algorithm results were used, whereas research into the possibilities of the use of the DISCO in a dimensional way to assess severity of symptoms can also be valuable, since ASDs are more and more considered to form a spectrum with different levels of severity (APA, 2010; Lord \& Bishop, 2010). In that respect, the DISCO is a useful addition to other autism specific clinical and research instruments, as the DISCO covers many more areas of development and behavior, also aspects of ASD which are not included in the official diagnostic criteria (Wing et al., 2002).

\section{References}

American Psychiatric Association [APA] (2010). DSM-V Development: Proposed revisions. Available at http://www.dsm5.org/ProposedRevisions/Pages/proposedrevision.aspx?rid=94..

Berument, S. K., Rutter, M., Lord, C., Pickles, A., \& Bailey, A. (1999). Autism Screening Questionnaire: Diagnostic validity. The British Journal of Psychiatry, 175, 444-451.

Bhaumik, S., Tyrer, F., Barrett, M., Tin, N., McGrother, C. W., \& Kiani, R. (2010). The relationship between carers' report of autistic traits in clinical diagnoses of autism spectrum disorders in adults with intellectual disability. Research in Developmental Disabilities, 31, 705-712.

Bishop, S. L., Luyster, R., Richler, J., \& Lord, C. (2008). Diagnostic assessment. In: K. Charwarska, A. Klin, \& F. R. Volkmar (Eds.), Autism spectrum disorders in infants and toddlers: Diagnosis, assessment, and treatment (pp. 23-49). New York, NY: Guilford Press.

Chandler, S., Charman, T., Baird, G., Simonoff, E., Loucas, T., Meldrum, D., Scott, M., \& Pickles, A. (2007). Validation of the Social Communication Questionnaire in a population cohort of children with autism spectrum disorders. Journal of American Academic Child and Adolescent Psychiatry, 46, 1324-1332.

Corsello, C., Hus, V., Pickles, A., Risi, S., Cook, E. H. Jr., Leventhal, B. L., \& Lord, C. (2007). Between a ROC and a hard place: Decision making and making decisions about using the SCQ. Journal of Child Psychology and Psychiatry, 48, 932-940.

De Bildt, A., Sytema, S., Ketelaars, C., Kraijer, D., Mulder, E., Volkmar, F., \& Minderaa, R. (2004). Interrelationship between Autism Diagnostic Observation Schedule-Generic (ADOS-G), Autism Diagnostic Interview-Revised (ADI-R), and the Diagnostic and Statistical Manual of Mental Disorders (DSM-IV-TR) classification in children and adolescents with mental retardation. Journal of Autism and Developmental Disorders, 34, 129-137. 
De Bildt, A., Sytema, S., Ketelaars, C., Kraijer, D., Volkmar, F., \& Minderaa, R. (2003). Measuring pervasive developmental disorders in children and adolescents with mental retardation: A comparison of two screening instruments used in a study of the total mentally retarded population from a designated area. Journal of Autism and Developmental Disorders, 33, 595-603.

Evers, A., Braak, M. S. L., Frima, R. M., \& Van Vliet-Mulder, J. C. (2009). Commissie Testaangelegenheden Nederland Documentatie [Committee for Psychometric Evaluation of Psychological Tests of the Dutch Institute of Psychologists - Documentation]. Amsterdam, NL: Boom Test Uitgevers.

Filipek, P. A., Accardo, P. J., Baranek, G. T., Cook, E. H. Jr., Dawson, G., Gordon, B., ... \& Volkmar, F. R. (1999). The screening and diagnosis of autistic spectrum disorders. Journal of Autism and Developmental Disorders, 29, 439-484.

Gotham, K., Risi, S., Pickles, A., \& Lord, C. (2007). The Autism Diagnostic Observation Schedule: Revised algorithms for improved diagnostic validity. Journal of Autism and Developmental Disabilities, 37, 613-627.

Gotham, K., Risi, S., Dawson, G., Tager-Flusberg, H., Joseph, R., Carter, A., ... \& Lord, C. (2008). A replication of the Autism Diagnostic Observation Schedule (ADOS) revised algorithms. Journal of the American Academy of Child \& Adolescent Psychiatry, 47, 642-651.

Gray, K. M., Tonge, B. J., \& Sweeney, D. J. (2008). Using the Autism Diagnostic Interview-Revised and the Autism Diagnostic Observation Schedule with young children with developmental delay: Evaluating diagnostic validity. Journal of Autism and Developmental Disorders, 38, 657-667.

Leekam, S. R., Libby, S. J., Wing, L., Gould, J., \& Taylor, C. (2002). The Diagnostic Interview for Social and Communication Disorders: Algorithms for ICD-10 Childhood Autism and Wing \& Gould autistic spectrum disorder. Journal of Child Psychology and Psychiatry, 43, 327-347.

Lord, C., \& Bishop, S. L. (2010). Autism spectrum disorders: Diagnosis, prevalence, and services for children and families. Social Policy Report, 24, 1-27.

Lord, C., Risi, S., Lambrecht, L., Cook, E. H. Jr., Leventhal, B. L., DiLavore, P. C., ... \& Rutter, M. (2000). The Autism Diagnostic Observation Schedule - Generic: A standard measure of social and communication deficits associated with the spectrum of autism. Journal of Autism and Developmental Disorders, 30, 205-223.

Lord, C., Rutter, M., DiLavore, P. C., \& Risi, S. (1999). Autism Diagnostic Observation Schedule (ADOS). Los Angeles, CA: Western Psychological Services.

Lord, C., Rutter, M., \& Le Couteur, A. (1994). Autism Diagnostic Interview-Revised: A revised version of a diagnostic interview for caregivers of individuals with possible pervasive developmental disorders. Journal of Autism Developmental Disorders, 24, 659-685.

Nygren, G., Hagberg, B., Billstedt, E., Skoglund, A., Gillberg, C., \& Johansson, M. (2009). The Swedish version of the Diagnostic Interview for Social and Communication Disorders (DISCO-10). Psychometric properties. Journal of Autism and Developmental Disorders, 39, 730-741.

Posserud, M., Lundervold, A. J., Lie, S. A., \& Gillberg, C. (2009). The prevalence of autism spectrum disorders: impact of diagnostic instrument and non-response bias. Social Psychiatry and Psychiatric Epidemiology, 45, 319-327.

Rutter, M., Bailey, A., \& Lord, C. (2003). SCQ: Social Communication Questionnaire. Los Angeles, CA: Western Psychological Services.

Schlichting, J. E. P. T., Van Eldik, M. C. M., Spelberg, H. C., Van der Meulen, S., \& Van der Meulen, B. F. (1995). Schlichting Test voor Taalproductie. [Schlichting Test for Language production]. Nijmegen, NL: Berkhout.

Snow, A. V., \& Lecavalier, L. (2008). Sensitivity and specificity of the Modified Checklist for Autism in Toddlers and the Social Communication Questionnaire in preschoolers suspected of having pervasive developmental disorders. Autism, 12, 627-644.

Tellegen, P. J., Winkel, M., Wijnberg-Williams, B. J., \& Laros, J. A. (1998). Snijders-Oomen Niet-verbale intelligentietest, SON-R 21/2-7: handleiding en verantwoording. [Snijders-Oomen Non-verbal intelligence test, SON-R 21/2-7: Manual]. Lisse, NL: Swets \& Zeitlinger.

Van Berckelaer-Onnes, I. A., Noens, I., \& Dijkxhoorn, Y. (2008). Diagnostic Interview for Social and Communication Disorders: Nederlandse Vertaling [Diagnostic Interview for Social and Communication Disorders: Dutch Translation]. Leiden, NL: Universiteit Leiden.

Van Eldik, M. C. M., Schlichting, J. E. P. T., Spelberg, H. C., Van der Meulen, S., \& Van der Meulen, B. F. (1995) Reynell Test voor Taalbegrip [Reynell Test for Language comprehension]. Nijmegen, NL: Berkhout.

Vig, S. \& Jedrysek, E. (1999). Autistic features in young children with significant cognitive impairment: Autism or mental retardation? Journal of Autism and Developmental, 29, 235-248.

Warreyn, P., Raymaekers, R., \& Roeyers, H. (2004). SCQ - Vragenlijst Sociale Communicatie [Social Communication Questionnaire]. Destelbergen, BE: SIG.

Wiggins, L. D., Bakeman, R., Adamson, L. B., \& Robins, D. L. (2007). The utility of the Social Communication Questionnaire in screening for autism in children referred for early intervention. Focus on Autism and Other Developmental Disabilities, 22, 33-38.

Wing, L. (2006). Diagnostic Interview for Social and Communication Disorders (11th ed.). Bromley, UK: Centre for Social and Communication Disorders.

Wing, L., Leekam, S. R., Libby, S. J., Gould, J., \& Larcombe, M. (2002). The Diagnostic Interview for Social and Communication Disorders: Background, inter-rater reliability and clinical use. Journal of Child Psychology and Psychiatry, 43, 307-325. 
Witwer, A. N., \& Lecavalier, L. (2007). Autism screening tools: An evaluation of the Social Communication Questionnaire and the Developmental Behaviour Checklist-Autism Screening Algorithm. Journal of Intellectual \& Developmental Disability Research, 32, 179-187. 